\title{
23. Preoperative and Potoperative RI-Gisternogram in Hydrocephalus
}

\author{
-Special Reference to Ventricular Reflux- \\ Yoshitaka Nakada, Yutaka Maki, Morimasa Kono, \\ Tadao Nose and Susumu Miner
}

\section{Experimental Hydrocephalus Introduced by Laminaria}

\author{
Akihiko Hrrayama. Katsuji Taomoto and Satoru Matsumoto \\ Department of Neurosurgery, Kitano Hospital
}

\section{Surgical Approach to Microcephaly}

Toshio Beppu*, Mizuo Kagawa, Shigeru Sento, Hiroshi Kono, Kuniaki Matsumori, Tamotsu OsugI Toyoaki Shinohara, Nobuko Ogawa, Koichi Kitamura and Ryoho Okada**

* Tokyo Women's Medical College Neurological Institute Department of Neurosurgery

**National Children's Hospital Department of Neurology

In this paper, the authors investigated the possibility for surgical approach to the aquired microcephaly by a neuroradiological methods, especially by the cisternography. Our observations dealed with 22 cases of microcephalus (aquired type were obtained in 17 cases), aged from 0 to 7 years (about half of them, 1-2 years old), consisted of 10 males and 12 females.

Head circumference was measured below 2 standard deviation from mean length of normal head growth by Nellhaus. Plain X-ray examination, PEG, PVG and CAG were performed in all cases in order to check up closure of sutures, space occupying lesions, dilation of CSF system, and other pathological changes except for 3 chronic subdural hematomas. Furthermore, to the purpose of investigation of CSF hydrodynamic change, cisternography by ${ }^{99 \mathrm{~m}} \mathrm{Tc}$ and ${ }^{131}$ IHSA was also carried out in 8 cases of acquired microcephalus.

In the almost all cases of acquired types, demonstrable symmetrical dilatation 
of the lateral ventricles were visualized moderately in contrast with little dilatation of the genetic types. On cisternogram, delayed RI distribution rate from the spinal subarachnoidal space to the parasagittal areas was appeared and also asymmetrical abnormal RI concentration was visualized on the intracranial cavities in 5 cases.

In another 7 cases of the acquired microcephalus, RI clearance were measured by means of the direct injection of ${ }^{99 \mathrm{~m}} \mathrm{Tc}$ to the anterior horn of the lateral ventricle monitered by Dina Camera Scientigraph continuosusly.

Consequently, in 5 out of 7 cases, retention rate of CSF flow from the lateral ventricle to the basal cisterns revealed more than $43 \%$ which was exactly distinguishable from the normal level (less than 25\%). Therefore, this fact seemed to be important and informative of us to understand the nature of the acquired microcephalus. The ventriculo-atrial shunt was done in 6 cases for the purpose of repair of CSF circulating disturbance.

The postoperative 3 cases resulted in disappearance of clinical convulsion and elimination of spastic paraplegia. Our experience was small but satisfactory. It is believed for us that this results in our observation suggested some possibility of operative indication for these microcephaly and cisternography might be much value as clinical diagnostic aid.

\title{
26. Lumbosacral Lipoma in Ghildren
}

\author{
Kazuho ZinnaI* and Kazuo Yamaguchi** \\ *Division, Orthopedic Surgery, Kanagawa Children \\ Medical Center \\ **Neurosurgical Department, Yokohama University \\ School of Medicine
}

Total 46 cases of spina bifida who were examined at Kanagawa Children Medical Center from the period of May, 1970 to April, 1971, have been selected for those having lumbosacral lipoma into 7 cases and 3 out of these 7 have gone under the surgery.

The age span of these 7 children is from 3 months' old to 5 years' old and all children are male. All subcutaneous tumors consisted of elastic soft material were palpated on the middline of the lumbosacral region.

The skin over the tumor revealed a dimpling in 3 cases, and 3 cases showed portwine type of nevus, and cartilaginous material was felt in the tumor in two cases.

Five cases showed motor disturbance on lower extremity. Also bowel and bladder deficit were recognized in 5 cases. One case showed skin paresthesia in the dermatome region of $\mathrm{Sl}$ on both legs. One case revealed there was no remarkable neurological signis. X-ray studies in simple and laminography performed for all cases, disclosed were spina bifida and widening of bone canal. Myelography showed shadow 